\title{
17 (comunitania)
}

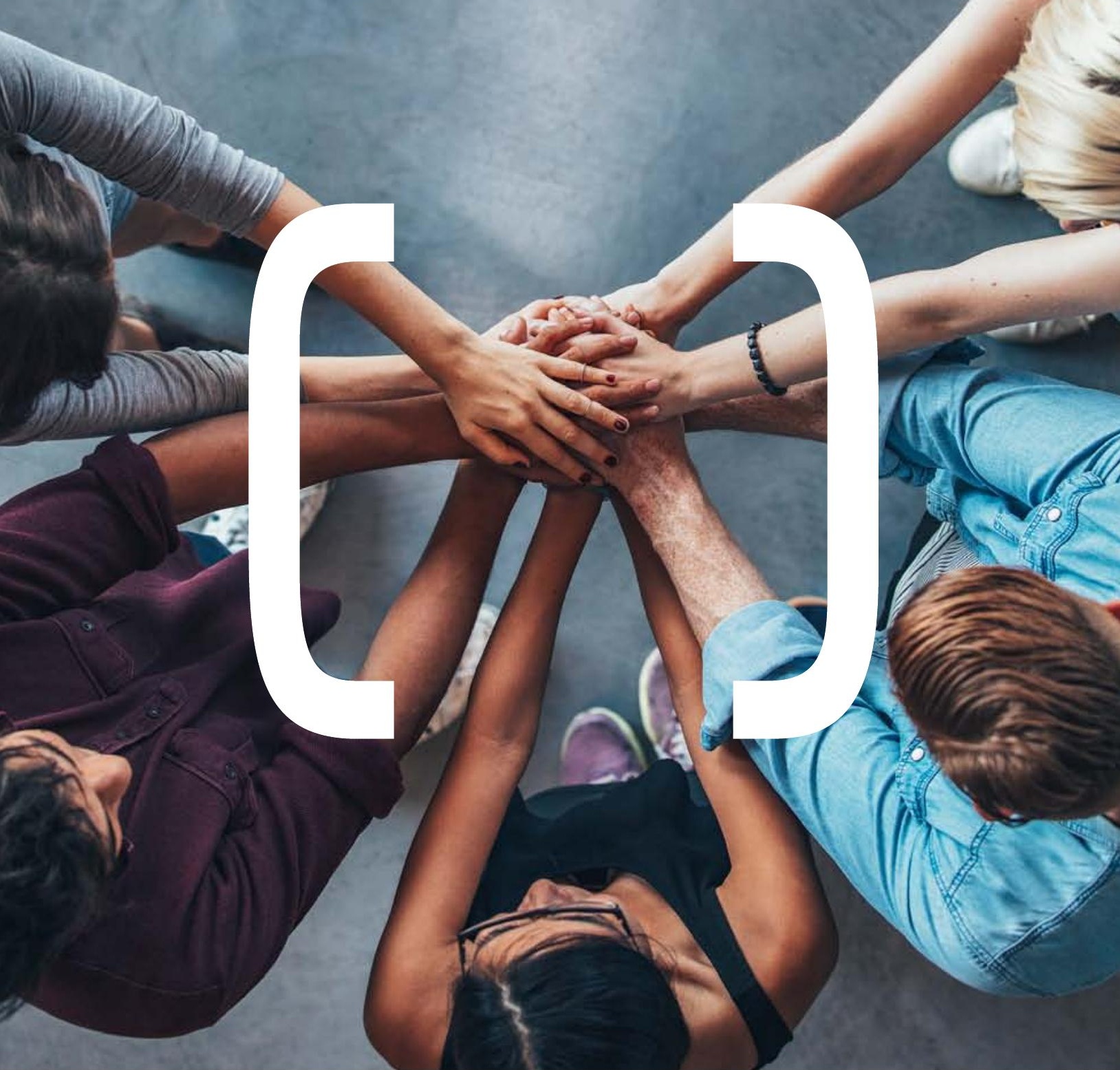

VICTORIA BOGINO-LARRAMBEBERE | CARLA CUBILLOS-VEGA | SOFIA DEDOTSI | VASILIOS IOAKIMIDIS | DIMITRA-DORA TELONI ISABEL MARTíNEZ SÁNCHEZ | ELISA ISABEL GÓMEZ VALLEJO | ROSA GOIG MARTÍNEZ | MARÍA ASUNCIÓN LILLO BENEYTO ANA MARÍA LÓPEZ-NARBONA | ELISA ESTEBAN CARBONELL | NURIA DEL OLMO VICÉN | CARLOS GÓMEZ BAHILLO 


\title{
Servicio de intervención familiar. De la normativa a la implementación: el caso de Aragón
} Family Intervention Service. From the regulation to the implementation: the case of Aragón

\author{
Elisa Esteban Carbonell, Nuria Del Olmo Vicén ${ }^{* *}$ Y Carlos Gómez Bahillo*** \\ * Universidad de Zaragoza. estecar@unizar.es \\ ** Universidad de Zaragoza.nolmov@unizar.es \\ *** Universidad de Zaragoza. cgomez@unizar.es
}

\begin{abstract}
:
The following article introduces, from an institutional perspective, the implementation differences of the family intervention service in Aragon. The research is based on the information extracted from the official online portals of the different administrations involved (regions, commonwealths and municipalities). The main results show territorial inequalities in this service -which provides psychoeducational support to families who are in a situation of crisis, risk or vulnerability-. More specifically, a comparative analysis of the different services or programs is carried out with respect to the denomination, the intervention purpose, the target population, the coexistence of several programs aimed at families, the support provided to the family and the strategies used by professionals. This study aims to highlight the inequalities that exist, within a territory, regarding the implementation of the service.
\end{abstract}

Keywords: imagen institucional, intervención, familia, desigualdades, territorio.

\section{Resumen:}

El presente artículo expone un análisis de las diferencias respecto a la implementación del servicio de intervención familiar en Aragón desde la perspectiva de la imagen institucional. En este sentido, a partir de la información extraída de los portales oficiales online de las distintas administraciones implicadas (comarcas, mancomunidades y municipios) en la gestión de dicho servicio, se revelan las desigualdades territoriales respecto a un servicio que brinda apoyos psicoeducativos a las familias que se encuentran en situación de crisis, riesgo o vulnerabilidad. Más concretamente, se lleva a cabo un análisis comparativo del contenido de los diferentes servicios o programas ofrecidos respecto a la denominación, la finalidad que persigue la intervención, la población destinataria, la coexistencia de varios programas o servicios dirigidos a familias, los apoyos brindados a la familia y las estrategias utilizadas por los profesionales. Este estudio pretende evidenciar las desigual- 
dades que existen en un territorio respecto a la intervención de un servicio desde la imagen institucional que ofrecen los portales oficiales.

Palabras clave: institutional image, intervention, family, inequalities, territory.

\section{Article info:}

Received: 04/03/2018 / Received in revised form: 19/01/2019

Accepted: 25/01/2019 / Published online: 29/01/2019

DOI: http://dx.doi.org/10.5944/comunitania.17.7

\section{Introducción}

La transición a la parentalidad supone un acontecimiento de suma importancia en el desarrollo evolutivo de toda persona y ésta puede variar en función de características personales, sociales, culturales y de otra índole. Apoyar que ésta se lleve a cabo de modo satisfactorio, como señala Hidalgo (1998, citado por Martín-Quintana, et al. 2009: 121), "contribuye positivamente a la madurez personal de los que la realizan", sin embargo, no es una tarea fácil hoy en día debido a diversas circunstancias, como señalan varios autores (Máiquez, Rodrigo, Capote, \& Vermaes, 2000; Rodríguez, Máiquez, Martín, \& Byrne, 2008).

En primer lugar, Martín-Quintana, et al. (2009), considera que la parentalidad se ha visto modificada por los rápidos y profundos cambios históricos y sociales. La función de ser padres se ha diversificado debido, por una parte, a la multiplicidad de formas de convivencia que existen actualmente $y$, por otra, a la redefinición de los roles de género en el interior de la familia basados en principios más igualitarios tanto en lo que respecta a las relaciones de pareja como a las relaciones padreshijos. Al mismo tiempo, los padres no son los únicos agentes educativos, dado que los hijos se encuentran inmersos en distintas esferas de la sociedad y coexisten con otros agentes educativos, lo que alimenta la idea de pérdida de poder educativo de los padres sobre sus hijos.

En segundo lugar, "la parentalidad se construye y se desarrolla en distintos escenarios socioculturales" (Martín-Quintana, et al. 2009: 122), es decir, el entramado de relaciones en el que están inmersos los padres ayuda y favorece el aprendizaje de la tarea. Sin embargo, no consiste en una simple reproducción de los patrones aprendidos, sino que los padres la hacen propia al individualizarla y dotarla de significados personales.

En tercer lugar, "la parentalidad se desarrolla en diferentes niveles de actuación, desde el nivel estratégico de organización del escenario educativo hasta el nivel táctico de actuaciones concretas" (Martín-Quintana, et al. 2009: 122). Además, es importante contar con cierto nivel de flexibilidad para adaptarse a las circunstancias 
sobrevenidas, al mismo tiempo que es preciso reflexionar sobre cómo se está ejerciendo la parentalidad con el fin de poder modificarla. Por tanto, no funciona la simple repetición de patrones aprendidos, sino que la función de la parentalidad requiere un esfuerzo cognitivo.

Por último, la parentalidad necesita apoyos sociales para su correcto funcionamiento y aunque éstos (formales y/o informales) sean diferentes en función de las características y necesidades de las familias y del entorno en el que éstas se encuentran inmersas, todas las familias pueden necesitar apoyos para desarrollar sus responsabilidades parentales en algún momento de su vida, sobre todo por causa de crisis familiar o de circunstancias sobrevenidas.

En este sentido, estos apoyos no sólo deben procurarse con un fin terapéutico sino con un fin preventivo y de promoción del desarrollo de cada uno de los miembros de la familia, lo que es clave para adoptar un enfoque positivo en la intervención familiar, tal y como señalan Rodrigo López, Máiquez Chaves y Martín Quintana (2010: 9). Además, la población objeto de intervención no son únicamente las familias vulnerables o en riesgo, sino que va mucho más allá. Todas las familias en algún momento dado pueden requerir ayuda.

En consecuencia, el Consejo de Europa, ha desarrollado la Recomendación Rec (Consejo de Europa, 2006), sobre políticas de apoyo al ejercicio positivo de la parentalidad. La parentalidad positiva se refiere "al comportamiento de los padres fundamentado en el interés superior del niño, que cuida, desarrolla sus capacidades, no es violento y ofrece reconocimiento y orientación que incluyen el establecimiento de límites que permitan el pleno desarrollo del niño" (Rodrigo López, Máiquez Chaves, \& Martín Quintana, 2010: 11).

La recomendación europea va dirigida a los Estados miembros del Consejo de Europa para que éstos sean conscientes de la necesidad de diseñar estrategias que proporcionen apoyos a los padres para cumplir su responsabilidad en la crianza y educación de sus hijos. Más concretamente, los apoyos serán a través de: (a) las políticas familiares adecuadas que proporcionen las medidas legislativas, administrativas y financieras para crear las mejores condiciones posibles para la educación positiva; (b) la prestación de servicios de apoyo para padres tales como servicios de asesoramiento locales, líneas telefónicas de ayuda y programas educativos para padres; y (c) proporcionar servicios especializados para los padres en situación de riesgo para prevenir el desplazamiento innecesario de los niños del hogar familiar motivado por situaciones de maltrato. Crear las condiciones adecuadas significa también tomar medidas para eliminar los obstáculos a la parentalidad positiva, como son las políticas para promover una mejor conciliación de la vida familiar y laboral (Rodrigo López, Máiquez Chaves, Martín Quintana, \& Rodríguez Ruíz, 2015: 34). 
Estos apoyos tienen que diseñarse y ejecutarse en la línea de apoyos directos a las familias a través de programas de parentalidad, pero también se debe reforzar a las comunidades para que éstas creen redes de apoyo. Se puede decir, por tanto, que el ejercicio de la parentalidad se desarrolla en varios niveles que van desde la esfera privada a la esfera pública (desde la propia tarea evolutiva a la co-responsabilidad de la comunidad).

Dejando de lado los apoyos informales, los apoyos formales deben ser proporcionados por expertos profesionales. Éstos constituyen el brazo ejecutor de un conjunto planificado y ordenado de políticas sociales de familia, donde los programas de parentalidad positiva son sólo un recurso más. Pueden ser programas de acceso universal donde tengan cabida multitud de familias, así como programas de acceso limitado, orientados a familias en situación de riesgo bio-psico-social. Los programas de acceso universal contribuyen a eliminar cualquier tipo de estigmatización que pudiera existir, más aún cuando la búsqueda de estos apoyos está en los servicios sociales. Y aunque son apoyos formales, una de las tareas de los expertos debe ser crear una buena red de apoyos informales para las familias, de modo que la participación de la comunidad es imprescindible. De lo que se trata es de dotar de herramientas eficaces para que las familias sean capaces de desarrollar la parentalidad positiva, es decir, empoderar a las familias con el fin de ser autónomas.

Sin embargo, ¿cómo se han materializado estos apoyos formales en nuestra región? ¿Podemos hablar de una política familiar que incluya el apoyo a la parentalidad?

\section{Programas de apoyo en el contexto de Aragón para familias en dificultad}

Aragón, como otras regiones de países del sur de Europa, se caracteriza por la ausencia de una política familiar explícita, es decir, un escaso desarrollo de medidas para las familias (Flaquer, 2003). Ante esta situación, los padres pasan a ser los principales responsables en la provisión de bienestar a sus hijos.

De alguna manera, se ha considerado el papel preponderante de la familia en la cobertura de necesidades de sus miembros como uno de los elementos importantes que caracterizan nuestro modelo de bienestar, como apuntan Moreno Mínguez (2005), Añón y Miravet, Naldini (2005), Naldini (2002), Saraceno (2003), Parella (2000), Flaquer (2003), y Moreno (2006), conocido como modelo mediterráneo o familista. El Estado tiene un carácter residual frente al protagonismo de la familia, cuyo rasgo principal es el escaso desarrollo de las politicas familiares o de apoyo a Ia familia.

A pesar de los cambios acaecidos en las últimas décadas en la sociedad española y aragonesa, todavía no se ha asistido al desarrollo de una extensa política fami- 
liar explícita. Esto provoca que la sociedad siga confiando en la familia como proveedora de bienestar. Nos encontramos, en este sentido, en un círculo vicioso. Ante un escaso desarrollo de políticas familiares, la familia responde a las necesidades de sus miembros, lo que a su vez le otorga un merecido reconomiento como proveedora de protección. Este hecho ha sido legitimado culturalmente, amortiguando la aparición de reivindicaciones sociales en pro de una política familiar (Saraceno, 2003).

En el contexto español, a pesar del escaso desarrollo de políticas familiares podemos diferenciar tres ámbitos de intervención dentro de la política familiar: intervención legal, intervención económica o compensación pública de cargas familiares e intervención mediante servicios sociales (Meil, 1995). Las dos primeras vienen abordadas por parte del estado central, mientras que las actuaciones por parte de los servicios sociales corresponden a las administraciones autonómicas y locales $y$, en el caso de Aragón, a las administraciones comarcales (art. 47 Ley 5/2009, de 30 de junio, de Servicios Sociales de Aragón).

El peso de cada una de las intervenciones no ha sido siempre el mismo. La intervención económica ocupó una posición privilegiada durante el régimen franquista, sin embargo se diluyó durante la transición política dando paso al desarrollo de una política de servicios sociales (Meil, 1995). Los servicios sociales muestran el ejemplo más visible de la política familiar española.

Siguiendo a Meil, a este hecho hay que añadir el creciente papel otorgado a las familias en la resolución de los problemas sociales. Frente a la intervención institucional, se tiende a promover el empoderamiento de la familia. Surge, en este sentido, un amplio abanico de servicios de apoyo a las familias en crisis, en riesgo de estarlo o en dificultad social de distinto tipo (servicio de respiro, formación, ayudas específicas, el acogimiento familiar, etc.), a fin de que puedan, efectivamente, asumir cargas familiares sin menoscabo de los derechos y del bienestar individuales (Meil, 1995: 71).

El momento clave en el desarrollo de programas de apoyo en el ejercicio de la parentalidad surge a comienzos del siglo XXI, a partir de la aprobación de la Recomendación Rec(2006)19 por parte del Comité de Ministros del Consejo de Europa. El objetivo es que los Estados reconozcan la importancia de la responsabilidad parental y la necesidad de que los padres tengan suficientes apoyos para cumplir con sus responsabilidades en la educación de sus hijos (Consejo de Europa, 2006).

Aunque la política familiar ha sido un asunto abordado desde instituciones europeas, hasta el momento la parentalidad positiva no ha sido incluida en la agenda política. La parentalidad positiva favorece el desarrollo de relaciones paterno-filiales beneficiosas y la optimización del potencial de desarrollo del niño (Consejo de Europa, 2006: 2). Las políticas públicas deben estar a la altura de las circunstancias e 
incorporar las demandas familiares surgidas como resultado de los rápidos y profundos cambios sociales.

Tanto las recomendaciones europeas sobre parentalidad positiva (Consejo de Europa, 2006), como los desarrollos posteriores a nivel nacional (Rodrigo López, Máiquez Chaves, \& Martín Quintana, 2010a, 2010b) han permitido, por un lado, aportar un lenguaje común que permite reflexionar de forma conjunta sobre el papel de la familia y el ejercicio de la parentalidad y, por otro, desarrollar recomendaciones prácticas sobre cómo articular los apoyos que las familias necesitan. En ambos casos, se destacan los programas psicoeducativos de formación y apoyo familiar como un vehículo idóneo para satisfacer las necesidades de apoyo en materia de parentalidad positiva (Jiménez \& Hidalgo, 2016).

La vigente ley de servicios sociales aragonesa -ley 5/2009 de 30 de junio de Servicios Sociales de Aragón- contempla el servicio de intervención familiar como un "conjunto de atenciones profesionales dirigidas a favorecer la convivencia y la integración social, fomentando la adquisición de habilidades básicas y hábitos de conducta, tanto en lo relativo a las capacidades personales como las relacionales, en situaciones de crisis, riesgo, o vulnerabilidad de cualquier miembro de la unidad de convivencia. Aporta una intervención técnica dirigida a orientar, asesorar y prestar apoyo cuando la estabilidad está amenazada o cuando no pueda garantizarse suficientemente la adecuada atención a algún miembro"(Departamento de Servicios Sociales y Familia, 2011: 15622). Y aunque implantada en toda la comunidad autónoma, todavía no cuenta con desarrollo legislativo propio.

El servicio de intervención familiar no es sí un programa concreto, y la interpretación del mismo varía considerablemente a lo ancho y largo del territorio aragonés, por lo tanto debemos preguntarnos si todas las familias tienen acceso a un mismo servicio a pesar de vivir en municipios distintos.

\section{Metodología}

A partir de la imagen institucional que ofrecen las distintas administraciones implicadas en su gestión, el estudio pretende comprobar si existen o no diferencias respecto a la implementación del servicio de intervención aragonés. Se trata de un análisis comparativo del contenido a partir de fuentes primarias oficiales.

Hay que tener en cuenta, como señala Corbetta (2010: 403) que "Ios documentos no suelen ser representaciones objetivas de la realidad institucional a la que se refieren, sino una representación oficial de la misma". A pesar de ello, los portales oficiales son las únicas unidades de análisis que unifican el acceso y, por lo tanto, nuestra principal fuente. Se ha extraído toda la información correspondiente a la "intervención familiar", más concretamente, qué programas o servicios se ofrecían a las familias. 
En este sentido, se realizó una búsqueda exhaustiva durante los meses de octubre y noviembre de 2017 para comprobar si dicho servicio se prestaba, bajo qué denominación y qué definición o concreción del mismo se tenía. La información recogida se ha segmentado en función de las siguientes variables a analizar: denominación, número de servicios/programas por centro, objeto de la intervención, población destinataria, finalidad, apoyos brindados a las familias, y herramientas o estrategias empleadas por los profesionales. Posteriormente se han codificado y analizado mediante SPSS con el fin de verificar si los individuos tienen acceso igual al mismo tipo de programa, es decir, destacar las diferencias si existen diferencias en las prestaciones que recibe el sujeto.

\section{Divergencias en la implementación del servicio de intervención familiar}

Situándonos en la perspectiva de la imagen institucional, se puede afirmar que existen grandes desigualdades respecto al servicio de intervención familiar, regulado por el Gobierno de Aragón y cuya implementación, en teoría, debería ser la misma para todo el territorio aragonés, es decir, aunque el servicio de intervención familiar cuenta con un respaldo normativo que garantiza, en teoría, su puesta en marcha de manera homogénea en toda la Comunidad Autónoma, la realidad es bien distinta. Nos encontramos con 41 "entidades públicas" diferentes (tabla 1) encargadas de su implementación. De estas 41 entidades públicas, tenemos 32 comarcas constituidas hasta la fecha. La comarca número 33 -donde se encuentra Zaragoza capital y municipios de su área metropolitana-, todavía no se ha constituido. Esta comarca eminentemente urbana, que concentra más de la mitad de la población total aragonesa, se organiza a partir de 3 mancomunidades (agrupaciones de municipios que gestionan diversas acciones), y 3 ayuntamientos con gestión propia (Zaragoza capital, Utebo y Fuentes de Ebro, y de éste último dependen dos municipios próximos a él). A las entidades señaladas, hay que añadir las ciudades deTeruel, Huesca y Calatayud que cuentan con una gestión propia, al tener las competencias sobre los servicios sociales al superar los 20.000 habitantes cada una de ellas (Ley 7/1985, de 2 de abril, Reguladora de las Bases del Régimen Local).

Ante esta complejidad territorial, uno de los objetivos de este estudio se orienta a observar cómo se articula o se concreta, en cada una de estas entidades, el servicio de intervención familiar.

En primer lugar, ¿qué información del servicio está disponible en los portales oficiales? Mientras que algunas comarcas, mancomunidades o municipios cuentan con una página web de acceso fácil donde el ciudadano encuentra sin grandes problemas el área de acción social, bienestar social o servicios sociales y los servicios, prestaciones o programas que se ofrecen, en otros la búsqueda se vuelve complicada, e incluso insatisfactoria, como sucede en la comarca de La Litera (Huesca), la comarca de Hoya de Huesca (Huesca), la comarca de Bajo Cinca (Huesca), la comar- 
ca de Tarazona y el Moncayo (Zaragoza) y el Ayuntamiento de Calatayud (Zaragoza). En estos casos, no podemos afirmar con rotundidad la no existencia del servicio, pero sí la no existencia en los correspondientes portales oficiales. No aparece a pesar de que, en algunos casos, sí lo hacen otros servicios o prestaciones ofertadas por los servicios sociales.

Aunque los servicios sociales están presentes en alguno de los portales de las entidades señaladas anteriormente, la información presentada no está con un fin divulgativo/informativo sino más bien responde a lógicas organizativas. Por ejemplo, en el caso del Ayuntamiento de Calatayud entre los servicios municipales encontramos "Bienestar Social" y dentro de éste grandes programas sociales sin seguir un criterio único. Es decir, un programa respecto a un colectivo -atención al inmigrante-, otro respecto a una problemática -prevención de drogodependencia-, otro en relación al objeto de la intervención -ayuda a domicilio-, y el resto siguiendo probablemente otros criterios -información y orientación, prevención e inserción social, cooperación y solidaridad-, al no encontrar en estos 5 casos información, se ha procedido a excluirlos del resto del análisis. Por tanto, el análisis se circunscribe a las 36 entidades que cuentan con alguna información en la página web.

En segundo lugar, ¿El servicio de intervención familiar recibe la misma denominación en cada una de las entidades? Si acudimos a la legislación autonómica, no hay ningún tipo de duda, puesto que tanto la Ley 5/2009 de 30 de junio de Servicios Sociales de Aragón como el Decreto 143/2011, de 14 de junio, del Gobierno de Aragón, por el que se aprueba el Catálogo de Servicios Sociales de la Comunidad Autónoma de Aragón ofrecen una descripción del mismo. La realidad nuevamente se contrapone a la normativa, en el sentido que no todas las "entidades públicas" ofrecen el servicio guardando la misma denominación.

De las 36 entidades públicas analizadas, únicamente 13 mantienen la denominación recogida en la legislación autonómica, 23 optan por ofrecer servicios bajo otras denominaciones. De estas 23, 7 mantienen denominaciones próximas a la original y 16 apuestan por denominaciones variadas; entre las 13 entidades que optan por la misma denominación que la original, la mayoría se sitúan en la provincia de Teruel. Por otra parte, encontramos 7 entidades públicas que, si bien no se ciñen estrictamente a la denominación, sí que se aproximan mucho. En este sentido, encontramos algunas que añaden aparentemente algún servicio más, o bien especifican la intervención a realizar, como es el caso de la Comarca Comunidad de Calatayud (Servicio de Intervención y Mediación Familiar); otras que prefieren enmarcar este tipo de intervención bajo un programa en lugar de un servicio, es el caso, entre otros, de los municipios pertenecientes a la Mancomunidad de la Ribera Izquierda del Ebro (Programa de Intervención Familiar); y otros que simplemente ofertan el servicio de manera general, como la Comarca del Bajo Aragón-Caspe que lo denominada "intervención familiar". 
Las restantes 16 entidades públicas (La Jacetania, Alto Gállego, Ayuntamiento de Huesca, Cinca Medio, Cinco Villas, Campo de Borja, Comarca del Aranda, Campo de Daroca, Ribera Baja del Ebro, Campo de Belchite, Bajo Martín, Mancomunidad Ribera Bajo Huerva, Ayuntamiento de Fuentes de Ebro, Mancomunidad del Bajo Gállego, Ayuntamiento de Utebo y Ayuntamiento de Zaragoza)optan por ofrecer servicios bajo denominaciones bien distintas y variadas. Así, encontramos "Servicio de Apoyo Familiar", "Servicio de Terapia Familiar para familias con hijos adolescentes" (ambas pertecientes a la Comarca La Jacetania -en la provincia de Huesca-), "Programa de apoyo a las familias y unidades de convivencia", "Atención individual y/o familiar", "Servicio para menores", "Programa de Educación Familiar", "Escuela de Familias", "Apoyo a la Unidad de Convivencia y Ayuda a Domicilio", "Programa de Prevención e Inserción Social", "Servicio de Orientación socioeducativa para familias y adolescentes", "Apoyo a las familias y a las diferentes unidades de convivencia", "Apoyo social, educativo, a la estructura y dinámica familiar", "Programa de Escuela de Padres y Madres", "Programa de Apoyo Familiar", "Servicio de apoyo a familias", "Escuela de Madres", "Apoyo a la unidad familiar", “Escuela de Padres" y "Programa de Apoyo a la Familia".

Llegados a este punto conviene señalar la coexistencia, en 5 comarcas, municipio y 1 mancomunidad, de varios servicios o programas dirigidos a la intervención con familias, lo que muestra la importancia que dicha entidad pública otorga a la familia. Así, podemos encontrar intervenciones centradas en grupos de población (adolescentes), intervenciones concretas (mediación, terapia, etc.), así como intervenciones que responden a lógicas específicas (escuela de familias, de padres, de madres, etc.).

La multiplicidad de denominaciones nos conduce directamente al tercer punto de nuestro análisis, ¿todos ofrecen el mismo tipo de intervención? No todas las entidades locales proporcionan la misma información. Tan sólo la Comarca La Jacetania ofrece normativa propia, más concretamente, un reglamento municipal que regula un gran programa denominado "Programa de Apoyo a la Unidad de Convivencia" en el que está inserto el "Servicio de Apoyo Familiar". El resto de "entidades públicas" no muestran normativa propia. Esto no quiere decir, nuevamente, que no exista pero sí da muestras de los déficits respecto a la transferencia de información y disponibilidad de la misma en los portales oficiales.

La no existencia de normativa no conlleva directamente la no existencia de información o al menos, de una descripción del servicio/programa prestado. Bien es cierto, que en algunas ocasiones nos encontramos únicamente con el nombre del servicio sin especificar más sobre él. Ante esto, nos preguntamos, ¿cómo sabe el ciudadano si puede ser potencial participante/usuario del mismo? De las 36 entidades hasta ahora analizadas, 8 sólo proporcionan la denominación del servicio prestado, por tanto el análisis posterior se ha efectuado sobre las 28 entidades que ofrecen una descripción. 
El Servicio de Intervención Familiar, tal y como viene definido en el Catálogo de Servicios Sociales de Aragón y concretado algo más en el portal oficial del Gobierno de Aragón, va dirigido a familias que se encuentran en situación de crisis, riesgo o vulnerabilidad. De los 28 casos que cuentan con descripción, encontramos que 13 de ellos mantienen que dicha intervención se dirije a familias en situación de crisis, 14 a familias en situación de riesgo y, 11 a familias en situación de vulnerabilidad. Cabe añadir la adición de "situación de necesidad" presente en un único caso.

El uso de estos cuatro conceptos (crisis, riesgo, vulnerabilidad y necesidad) no excluyentes próximos entre sí dentro del ámbito de la intervención social, pero con connotaciones y realidades bien diversas, pone en relieve la necesidad de definir y acotar el perfil de familias usuarias, participantes o beneficiarias del programa. Además, este punto podría constituir un análisis en sí mismo tratando de desvelar qué sentido y significado se le da a cada uno de estos conceptos y cómo se traduce en términos de intervención.

Otro aspecto importante es señalar qué tipo de intervención se lleva a cabo (tabla 2). El Gobierno de Aragón únicamente afirma que es un conjunto de "atenciones profesionales" y que aporta una "intervención técnica". Ambos conceptos vagamente presentes. 4 casos del total de 28 que ofrecen información señalan "atenciones profesionales", y 5 "información técnica". Sin embargo, el concepto de "intervención socioeducativa" no reflejado en el servicio "marco" aparece en 11 de los 28 casos. A pesar que estamos en un plano teórico, gracias a este último término podemos pensar en cómo se implementa el servicio o programa o qué perspectiva adopta, puesto que los conceptos ofrecidos por el Gobierno de Aragón no permiten conocer qué tipo de intervencion se ofrece.

¿Qué finalidad persigue este tipo de intervenciones según la información ofrecida en los portales oficiales? Son muchas y muy variadas y, en ocasiones, demasiado generales, lo que impide conocer con exactitud en qué consiste la intervención. "Favorecer la convivencia y la integración social" es el objetivo más frecuente, de hecho se puede encontrar en 9 casos (Sobrarbe, Somontano de Barbastro, Valdejalón, Campo de Cariñena, Andorra-Sierra de Arcos, Jiloca, Sierra de Albarracín, Gúdar-Javalambre, y Comunidad de Teruel). Todo apunta, por tanto, que este tipo de intervención pretende que las familias se integren en su comunidad y en el conjunto de la sociedad de la forma más satisfactoria posible. Igualmente se encuentran objetivos similiares ("apoyo a la integración y socialización", "formación en hábitos convivenciales", "desarrollo de capacidades para la convivencia"), formulados de otra manera, pero que guardan una relación estrecha con el objetivo más frecuente.

Pero, si se tratan de intervenciones familiares ¿hay alguna finalidad centrada en la adquisición o mejora de las capacidades parentales? Rotundamente sí. Así, el más frecuente, aunque no quiere decir que sea muy extendido, dado que sólo está presente en 8 casos (Sobrarbe, Valdejalón, Ribera Alta del Ebro, Bajo Aragón, Bajo Mar- 
tín, Jiloca, Sierra de Albarracín, y Gúdar-Javalambre), es el objetivo "adquisición de habilidades básicas y hábitos de conducta". Sin embargo, puede parecer ambiguo o poco concreto. Conviene por tanto señalar otros objetivos que guardando una relación estrecha con éste, explicitan algo: "mantenimiento o mejora de habilidades personales y sociales", "autocuidado, cuidado y educación de los menores", "capacitar a los adutlos para el desempeño de roles parentales", "apoyo a la estructura familiar y a la dinámica de relación", "potenciar habilidades interrelacionales".

Tratándose de programas o servicios de parentalidad no podemos olvidarnos de la importancia que juega la prevención, tanto respecto a la aparición de factores en las familias usuarias, como dirigida a la comunidad en su conjunto. Es decir, en este caso podemos hablar de una prevención de tipo primario orientada a la sensibilización de la comunidad. En este sentido, podemos citar los siguientes objetivos relacionados con la prevención: "prevenir causas que generan exclusión social", "prevenir aparición procesos de marginación", "sensibilizar a la población", "evitar actitudes y comportamientos de rechazo social", "prevenir situaciones de riesgo". Hablamos por tanto de intervenciones de tipo comunitario, sin embargo, sólo 3 casos contemplan el carácter comunitario en sus intervenciones.

Otro principio importante de la intervención con las familias es la promoción o, lo que lo mismo, empoderar a las familias y dotarles de herramientas para que ellas sean capaces de resolver las dificultades con las que se encuentren. Por un lado, el servicio "marco" incide en quehay que"orientar, asesorar y dar apoyo a la familia o unidad de convivencia o a alguno de los miembros de la misma". Objetivo, en principio, lógico en tanto en cuanto se trata de una intervención familiar orientada a la adquisición y mejora de pautas parentales; sin embargo, escasamente presente en los casos analizados -únicamente en 5 descripciones aparece-. Por otro lado, ¿qué herramientas se les ofrece? ¿cómo se brindan los apoyos a las familias?. El servicio "marco" señala que las atenciones deben ir dirigidas en cuatro aspectos: orientaciones básicas para la organización económica y familiar; directrices de planificación de higiene familiar; formación en hábitos convivenciales; y apoyo a la integración y socialización. Aspectos presentes en algunas de las descripciones analizadas, más concretamente, en $5,4,4$ y 4 casos respectivamente.

Pero es posible encontrar en las diferentes descripciones más información respecto a qué técnicas o estrategias se utilizan en las intervenciones con las familias, más concretamente: "prestaciones y proyectos específicos", "orientación y mediación en relaciones familiares", "ayudas económicas", "apoyo social, psicológico y jurídico", "intervenciones asistenciales", "inserción sociolaboral", "sólo asesoramiento", "atenciones psicológicas". Todas ellas presentes en pocos y diferentes casos, lo que muestra las diferencias existentes respecto a la implementación del servicio.

Por último, es posible señalar que algunas intervencionesañaden más información en la descripción del servicio; por ejemplo, hay intervenciones que acotan la 
población destinataria (adolescentes entre 12 y 15 años), otras introducen la modalidad grupal y señalan que ésta sirve para que se lleve a cabo un "intercambio de experiencias y vivencias", otras concretan los profesionales encargados de la implementación del servicio/programa o simplemente señalan que hay "un equipo interdisciplinar", entre otros muchos aspectos.

\section{Conclusiones}

El análisis realizado pone en evidencia las diferencias territoriales respecto a un servicio garantizado y respaldado legislativamente. La intervención familiar dista mucho de ser la misma a lo largo de todo el territorio aragonés, al menos tras el minucioso estudio realizado. Más concretamente, diferencias respecto a la finalidad que persigue cada uno de los servicios o programas, las herramientas y el enfoque (psicoeducativo, asistencial, preventivo, etc.) empleados en su implementación, sería oportuno e interesante contrastar los resultados aquí presentados con los que se obtuvieran de un análisis de la práctica profesional, es decir, la diferencia entre la imagen proyectada y la intervención realizada. Sin embargo, este punto excede los objetivos del presente trabajo y será objeto principal en investigaciones posteriores.

En cualquier caso, conviene poner en valor la falta de homogeneidad en las denominaciones de los servicios o programas prestados que, aun sin ser un elemento crucial, puede ocasionar que el ciudadano considere que se tratan de diferentes programas o servicios. Ahondando en la descripción y tras un análisis exhaustivo es posible afirmar que, a grandes rasgos, el servicio es similar; sin embargo, es utópico pensar que el ciudadano realice tal análisis para comprobar que el servicio prestado en su comarca es similar a la comarca vecina. La homogeneización de los distintos tipos de intervenciones familiares es una prioridad, pero también lo es la transparencia y transferencia de información en aquellas "entidades públicas" en las que no ha sido posible realizar el análisis.

Al mismo tiempo se debe concretar qué se entiende por intervención familiar, qué finalidad persigue y qué herramientas o estrategias se van a emplear para alcanzar dicha finalidad. 


\section{TABLA 1. Entidades públicas}

\begin{tabular}{|c|c|c|}
\hline \multirow[t]{2}{*}{$\begin{array}{c}\text { Huesca } \\
\text { (11 entidades) }\end{array}$} & Comarcas & $\begin{array}{l}\text {-La Jacetania } \\
\text {-Alto Gállego } \\
\text {-Sobrarbe } \\
\text {-La Ribagorza } \\
\text {-La Litera } \\
\text {-Somontano de Barbastro } \\
\text {-Hoya de Huesca } \\
\text {-Cinca Medio } \\
\text {-Bajo Cinca } \\
\text {-Los Monegros }\end{array}$ \\
\hline & Ayuntamientos & -Ayuntamiento de Huesca \\
\hline \multirow[t]{3}{*}{$\begin{array}{c}\text { Zaragoza } \\
\text { (19 entidades) }\end{array}$} & Comarcas & $\begin{array}{l}\text {-Cinco Villas } \\
\text {-Tarazona y el Moncayo } \\
\text {-Campo de Borja } \\
\text {-Comarca del Aranda } \\
\text {-Valdejalón } \\
\text {-Comunidad de Calatayud } \\
\text {-Campo de Cariñena } \\
\text {-Campo de Daroca } \\
\text {-Ribera Alta del Ebro } \\
\text {-Ribera Baja del Ebro } \\
\text {-Campo de Belchite } \\
\text {-Bajo Aragón-Caspe }\end{array}$ \\
\hline & Mancomunidades & $\begin{array}{l}\text {-Mancomunidad de la Ribera Izquierda del Ebro } \\
\text {-Mancomunidad Ribera Bajo Huerva } \\
\text {-Mancomunidad Bajo Gállego }\end{array}$ \\
\hline & Ayuntamientos & $\begin{array}{l}\text {-Ayuntamiento de Calatayud } \\
\text {-Ayuntamiento Fuentes de Ebro } \\
\text {-Ayuntamiento de Utebo } \\
\text {-Ayuntamiento de Zaragoza }\end{array}$ \\
\hline \multirow[t]{2}{*}{$\begin{array}{c}\text { Teruel } \\
\text { (11 entidades) }\end{array}$} & Comarcas & $\begin{array}{l}\text {-Matarraña } \\
\text {-Bajo Aragón } \\
\text {-Bajo Martín } \\
\text {-Andorra-Sierra de Arcos } \\
\text {-Cuencas Mineras } \\
\text {-Jiloca } \\
\text {-Sierra de Albarracín } \\
\text {-Maestrazgo } \\
\text {-Gúdar-Javalambre } \\
\text {-Comunidad de Teruel }\end{array}$ \\
\hline & Ayuntamientos & -Ayuntamiento de Teruel \\
\hline
\end{tabular}

Fuente: Elaboración propia a partir de la información ofrecida en los portales oficiales. 
Tabla 2. Tipos de intervención según entidad y denominación

\begin{tabular}{|c|c|c|}
\hline Entidad pública & Denominación & Tipo de intervención \\
\hline $\begin{array}{l}\text { Comarca Somontano } \\
\text { de Barbastro }\end{array}$ & $\begin{array}{l}\text {-Servicio de Intervención } \\
\text { Familiar } \\
\text {-Programa de prevención e } \\
\text { inserción social }\end{array}$ & $\begin{array}{l}\text { Atenciones profesionales } \\
\text { Intervención técnica } \\
\text { Intervenciones socio-educativas }\end{array}$ \\
\hline $\begin{array}{l}\text { Comarca de Cinca } \\
\text { Medio }\end{array}$ & -Servicio para menores & Intervención socio-educativa \\
\hline $\begin{array}{l}\text { Comarca Los } \\
\text { Monegros }\end{array}$ & $\begin{array}{l}\text {-Servicio de intervención } \\
\text { familiar }\end{array}$ & Intervención técnica \\
\hline Comarca Cinco Villas & $\begin{array}{l}\text {-Programa de Educación } \\
\text { Familiar }\end{array}$ & Actuaciones profesionales \\
\hline $\begin{array}{l}\text { Comarca de } \\
\text { Comunidad de } \\
\text { Calatayud }\end{array}$ & $\begin{array}{l}\text {-Servicio de Intervención y } \\
\text { Mediación familiar }\end{array}$ & Intervención técnica \\
\hline $\begin{array}{l}\text { Comarca Campo de } \\
\text { Cariñena }\end{array}$ & $\begin{array}{l}\text {-Programa de Intervención } \\
\text { Familiar }\end{array}$ & Atenciones profesionales \\
\hline $\begin{array}{l}\text { Comarca Campo de } \\
\text { Daroca }\end{array}$ & $\begin{array}{l}\text {-Programa de Apoyo a la } \\
\text { Unidad de Convivencia y Ayuda } \\
\text { a Domicilio } \\
\text {-Programa de Prevención e } \\
\text { Inserción Social }\end{array}$ & Actuaciones socioeducativas \\
\hline $\begin{array}{l}\text { Comarca Ribera Alta } \\
\text { del Ebro }\end{array}$ & $\begin{array}{l}\text {-Intervención Familiar } \\
\text {-Servicio de Orientación } \\
\text { socioeducativa para familias y } \\
\text { adolescentes }\end{array}$ & Actuaciones psicoeducativas \\
\hline $\begin{array}{l}\text { Comarca Bajo } \\
\text { Aragón-Caspe }\end{array}$ & -Intervención familiar & $\begin{array}{l}\text { Atenciones psicológicas, } \\
\text { socioeducativas, técnicas y de } \\
\text { rehabilitación }\end{array}$ \\
\hline $\begin{array}{l}\text { Comarca Andorra- } \\
\text { Sierra de Arcos }\end{array}$ & $\begin{array}{l}\text {-Servicio de intervención } \\
\text { familiar }\end{array}$ & $\begin{array}{l}\text { Intervención profesional de los } \\
\text { técnicos }\end{array}$ \\
\hline $\begin{array}{l}\text { Comarca Cuencas } \\
\text { Mineras }\end{array}$ & $\begin{array}{l}\text {-Programa de intervención } \\
\text { familiar }\end{array}$ & $\begin{array}{l}\text { Atenciones o cuidados de carácter } \\
\text { personal, psicosocial y educativo, } \\
\text { doméstico y técnico }\end{array}$ \\
\hline $\begin{array}{l}\text { Comarca Comunidad } \\
\text { de Teruel }\end{array}$ & $\begin{array}{l}\text {-Servicio de Intervención } \\
\text { Familiar }\end{array}$ & Intervención profesional \\
\hline $\begin{array}{l}\text { Ayuntamiento de } \\
\text { Fuentes de Ebro }\end{array}$ & $\begin{array}{l}\text {-Servicio de Apoyo a familias } \\
\text {-Escuela de madres }\end{array}$ & $\begin{array}{l}\text { Apoyo de profesionales del Servicio } \\
\text { Social }\end{array}$ \\
\hline
\end{tabular}

Fuente: Elaboración propia a partir de la información ofrecida en los portales oficiales. 


\section{Referencias:}

Añón, M., \& Miravet, P. (2005). "Paradojas del familiarismo en el Estado del bienestar: mujeres y renta básica". Cuadernos de Relaciones Laborales, 23 (2): 101-121. Consulta el 10 de enero de 2017 (https://revistas.ucm.es/index.php/CRLA/article/view/ CRLA0505220101A)

Consejo de Europa. (2006). Recomendación Rec (2006) 19 del Comité de Ministros a los Estados Miembros sobre políticas de apoyo a la parentalidad positiva. Consejo de Europa.

Corbetta, P. (2010). Metodología y técnicas de investigación social. Madrid: McGraw-Hill.

Departamento de Servicios Sociales y Familia. (2011). "Decreto 143/2011, de 14 de junio, del Gobierno de Aragón, por el que se aprueba el Catálogo de Servicios Sociales de la Comunidad Autónoma de Aragón". Boletín Oficial de Aragón 127: 15602-15736. 30 de junio de 2011.

Flaquer, L. (2003). "Familia y Estado de Bienestar en la Europa del sur". Arbor, 174 (685): 195-220. Consulta el 6 diciembre 2016 (http://arbor.revistas.csic.es/index.php/arbor/article/view/633/639)

Jiménez, L., \& Hidalgo, M. V. (2016). "La incorporación de prácticas basadas en evidencias en el trabajo con familias: los programas de promoción de parentalidad positiva". Apuntes de Psicología, 34(2-3): 91-100. Consulta el 14 marzo 2017 (https://idus.us.es/xmlui/handle/11441/64443)

Máiquez, M., Rodrigo, M., Capote, C., \& Vermaes, I. (2000). Aprender en la vida cotidiana. Un programa experiencial para padres. Madrid: Visor.

Martín-Quintana, J. C., Byrne, S., Máiquez Chaves, M. L., Rodríguez Ruiz, B., Rodrigo López, M. J., \& Rodríguez Suárez, G. (2009). "Programas de Educación Parental. Intervención Psicosocial," 18(2): 121-133.

Meil, G. (1995). Presente y futuro de la política familiar en España. Reis(70), 67-90.

Moreno Mínguez, A. (2005). "Empleo de la mujer y familia en los regímenes de bienestar del sur de Europa en perspectiva comparadad. Permanencia del modelo del varón sustentador". Revista Española de Investigaciones Sociológicas. REIS (112/05): 131-163. Consulta el 12 diciembre 2016 (http://www.reis.cis.es/REIS/ssp/REIS.jsp?opcion=articulo\&ktitulo=2013\&autor=ALMUDENA+MORENO+M\%CDNGUEZ)

Moreno, L. (2006). "España y el bienestar mediterráneo." Unidad de Políticas Comparadas (Documento de trabajo 06-10).

Naldini, M. (2002). The Family in the Mediterranean Welfare States.Londres: Frank Cass.

Parella, S. (2000). "Las políticas familiares". En J. Adelantado, Cambios en el Estado del Bienestar. Políticas sociales y desigualdades en España. Barcelona: Icaria.

Rodrigo López, M. J., Máiquez Chaves, M. L., \& Martín Quintana, J. C. (2010a). La educación parental como recurso psicoeducativo para promover la parentalidad. Madrid: Federación Española de Municipios y Provincias.

- (2010b). Parentalidad positiva y políticas locales de apoyo a las familias. Madrid: Federación Española de Municipios y Provincias.

Rodrigo López, M., Máiquez Chaves, M., Martín Quintana, J., \& Rodríguez Ruíz, B. (2015). La parentalidad positiva desde la prevención y la promoción. En M. Rodrigo López, 
M. Máiquez Chaves, J. Martín Quintana, S. Byrne, \& B. Rodríguez Ruíz, Manual práctico de parentalidad positiva (págs. 25-43). Madrid: Síntesis.

Rodríguez, M., Máiquez, M., Martín, J., \& Byrne, S. (2008). Preservación Familiar: un enfoque positivo para la intervención con familias. Madrid: Pirámide.

Saraceno, C. (2003). Mutamenti della famiglia e politiche sociale in Italia. Bolonia: II Mulino. 


\section{comunitania}

REVISTA INTERNACIONAL DE TRABAJO SOCIAL Y CIENCIAS SOCIALES

INTERNATIONAL JOURNAL OF SOCIAL WORK AND SOCIAL SCIENCES

\section{ARTICULLOS/ARTICLES}

Los determinantes del desclasamiento social educativo. Un análisis sobre la cohorte de treintañeros con título superior en España / The Determinants of Social Educational Downclassing. An Analysis of the Thirty-Year-Old

Cohort in Spain

Victoria Bogino-Larrambebere

Págs 9-33

La formación en derechos humanos en el Trabajo Social. Evolución, propuestas y retos / Human Rights

Education in Social Work. Development, recommendations, and challenges

Carla Cubillos-Vega

Págs 35-58

La crisis de los Refugiados en Grecia: El desarrollo del racism. Respuestas antirracistas e implicaciones para el Trabajo Social / Refugee crisis in Greece: The development of racism, anti-racist responses and implications for social work

Sofia Dedotsi, Vasilios loakimidis y Dimitra-Dora Telon

Págs 59-69

El acoso escolar en educación secundaria: prevalencia y abordaje a través de un estudio de caso / Bullying in secondary education: prevalence and approach through a case study

Isabel Martínez Sánchez, Elisa Isabel Gómez Vallejo y Rosa Goig Martínez

Págs 71-91

Las percepciones de las personas usuarias sobre la intervención social del trabajo social / The perceptions of users about the social intervention of social work

María Asunción Lillo Beneyto

Págs 93-110

The social positioning of immigrants and the social order problem / El posicionamiento social de los

inmigrantes y el problema del orden social

Ana María López-Narbona

Págs 111-134

Servicio de intervención familiar. De la normativa a la implementación: el caso de Aragón / Family Intervention

Service. From the regulation to the implementation: the case of Aragón

Elisa Esteban Carbonell, Nuria Del Olmo Vicén y Carlos Gómez Bahillo

\section{RESENANAS/REVIEWIS}

Blanchard Giménez, M. (Coord.) (2014) Transformando la sociedad desde las aulas. Metodología de aprendizaje por proyectos para la innovación educativa en el Salvador / Transforming society from the classroom. Project learning methodology for educational innovation in El Salvador

(por M. a Ángeles Hernández Prados)

Págs 151-152

Watts, Beth y Fitzpatrick, Suzanne (2018) Welfare Conditionality / La condicionalidad en el estado del bienestar (por Domingo Carbonero Muñoz)

Págs 153-159

Cortina, Adela (2017). Aporofobia, el rechazo al pobre / Aporophobia, the rejection of the poor

(por Leonel Del Prado)

Págs 161-164

Paris A. Cabello-Tijerina \& Reyna L. Vázquez Gutiérrez. Cultura y Educación para la paz. Una perspectiva transversal / Culture and Education for Peace. A transversal perspective

(por Diego F. Yanten Cabrera) 